\title{
Correction to: IncRNA NONRATT021972 siRNA Decreases Diabetic Neuropathic Pain Mediated by the P2X3 Receptor in Dorsal Root Ganglia
}

\author{
Haiying Peng ${ }^{1} \cdot$ Lifang Zou $^{1} \cdot$ Jinyan Xie ${ }^{1} \cdot$ Hong Wu ${ }^{1} \cdot$ Bing Wu ${ }^{1} \cdot$ Gaochun Zhu ${ }^{1} \cdot$ Qiulan $\mathrm{Lv}^{1} \cdot \mathrm{Xi} \mathrm{Zhang}^{1} \cdot$

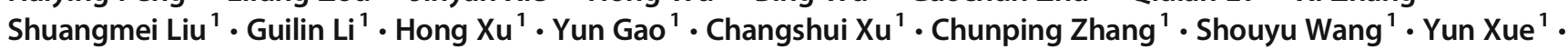 \\ Shangdong Liang ${ }^{1}$
}

Published online: 26 June 2019

(C) Springer Science+Business Media, LLC, part of Springer Nature 2019

\section{Correction to: Mol Neurobiol (2017) 54:511-523}

https://doi.org/10.1007/s12035-015-9632-1

In the original version of this article "lncRNA NONRATT021972 siRNA Decreases Diabetic Neuropathic Pain Mediated by the P2X3 Receptor in Dorsal Root Ganglia," which we have published in Mol Neurobiol (2017) 54:511-523. DOI https://doi.org/10.1007/s12035-015-9632-1, a correction is necessary.

We had carefully prepared the manuscript. However, due to the authors' carelessness, Fig. 1A images were incorrect. The statistical analysis histogram was not added, which was not consistent with the results described in the paper $(P<0.01)$. We repeated the experiment again, and the data accorded with the trend of the original results. In order to better display the expression of NONRATT021972 in neurons, Fig. 1A is corrected. The statistical analysis histogram has been added and corrected $(P<0.05)$.

Finally, we regret the errors on our part, as we did not catch the mistake during editing, and apologize for any confusion this may cause. We would like to extend our apologies to the readers and to the editorial board for the Molecular Neurobiology.

The online version of the original article can be found at https://doi.org/ 10.1007/s12035-015-9632-1

Shangdong Liang

liangsd@hotmail.com

1 Department of Physiology, Medical College of Nanchang University, Nanchang, Jiangxi 330006, People's Republic of China 


\section{A Control}
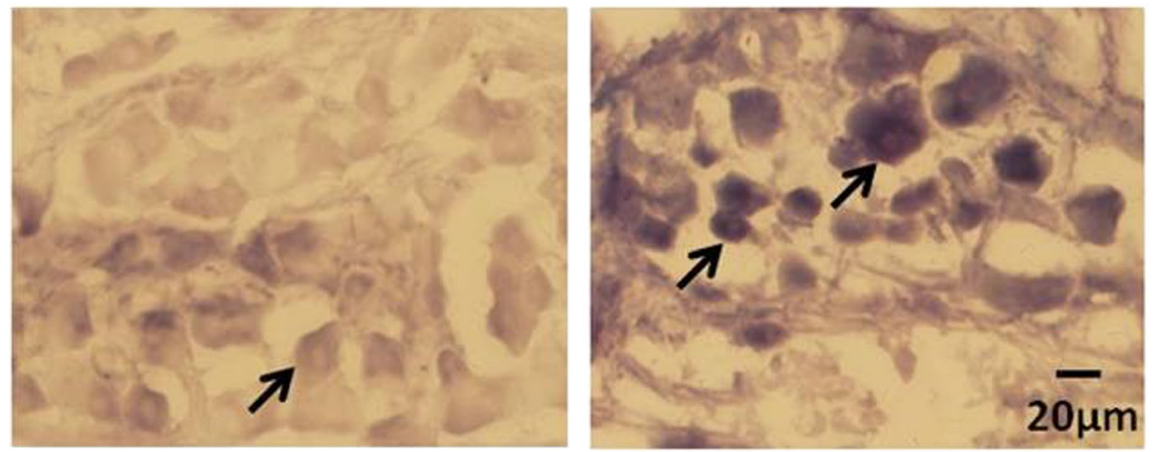

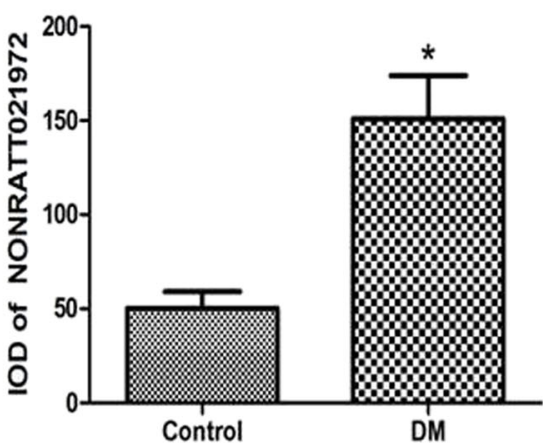

NONRATT021972 in DRG was tested using Q-PCR. The result revealed that the NONRATT021972 expression in T2DM DRG was higher compared to the control group ( $p<0.01, n=10$ for each group). The NONRATT021972 expression in T2DM rats treated with NONRATT021972 siRNA was significantly decreased compared with the T2DM group ( $p<0.01, n=10$ for each group). No difference in the DRG NONRATT021972 expression was found between the DM group and DM+ scramble siRNA group ( $p>0.05, n=10$ for each group). The corrected panels are shown below

Publisher's Note Springer Nature remains neutral with regard to jurisdictional claims in published maps and institutional affiliations. 